\title{
Analysis of perceptual characteristics of pain describing in words caused by occupational cervicobrachial disorder and similar disease ${ }^{1}$
}

\author{
AIKO SATOW 2,3 \\ Department of Psychology, Hamamatsu University School of Medicine, Hamamatsu, Shizuoka 431-31 \\ KATSUYA NAKATANI \\ College of General Arts and Science, Kinki University, Higashi-Osaka, Osaka 577
}

SHUNJI TANIGUCHI

Sugiyama Jogakuen Junior College, Chikusa-ku, Nagoya 464

In Research I, the sixteen words were supported as the pain descriptors by non-medical and medical subjects who have experienced these pains. Five perceptual characteristics (duration, interval, depth, area and intensity) of the pains described by the sixteen words were estimated by five-point scales and expressed in pentagon-profiles. Pains which were long duration and repeated frequently were deep and strong. Short and infrequent pains were shallow, weak and small. The long duration pains are deservedly intolerable than the short pains. The short pains are deservedly tolerable. The pentagon-profiles of the sixteen pains were ranked in order of " intolerability". In Research II, a Japanese version of MPQ was compiled and analyzed by the same procedure as those in Research I. The thirty-five words were supported and differentiated from emotional or evaluative words by the same subjects as participated in Research I. These words were also arranged by the rank order of intolerability. Reliability of the subjects' estimation of the pains was supported by results of the words which used common to both Researches I and II.

Key words: pentagon-profile, pain, tolerance, McGill, MPQ (McGill-Melzack Pain Questionnaire), percept, wrist, elbow, shoulder.

Wolff (1978) mentioned that, "In clinical pain, the physician's best and most valid index of the presence of pain is the

1 This research was supported by Grant-in-Aid for Scientific Research No. 59510055 (to the first author), Ministry of Education, Science and Culture, Japan. The research No. 59510055, contained two parts, (1) analysis of perceptual characteristics of the pain described by pain described words, and (2) design of apparatus and establishing the psychophysical method of measurement for the pain. The present paper offers results from the former (1).

2 We gratefully acknowledge the assistance of Mr. Roger Machin, Senior Lecturer, Chukyo University, in compiling an English version of the 25word list and the Japanese version of McGill Pain Questionnaire.

3 Correspondence and requests for reprints should be sent to Aiko Satow, Ph.D. patient's verbal report." In experimental research of pain, we think that subjects' verbal reports by pain-descriptive words are not only better indexes of present pains (subjects are perceiving it), but also indexes of prior pains (subjects have felt these. Their reports depend on their past experiences of the pains). Because in psychological experiments, actual pain-stimulation permitted ethically is only cutaneous stimulation, although there are many kinds of actual pain. For experimental research of these many kinds of pain, analysis of verbal reports of subjects who experienced these pains are better method of research, since actual pain-stimulations should not be applied to subjects. Studies of language of pain are in clinical research. Before presenting results of our work used 
pain descriptive words, brief review of researches concerning pain descriptive words are below.

Neuro-physiologists categorize three kinds pain; pricking pain, burning pain and aching pain. Pricking pain occurs by heat stimulation of the surface of the skin and then withdrawal reflex occurs. Burning pain occurs also by thermal stimulation which is below temperature of that for the pricking. Aching is located in deeper tissues than pricking and burning (Mountcastle, 1980). The other two categories are initial pain (first pain) and delayed pain (second pain). The latter has a dull or burning character (Schmidt 1978, pp. 111-113). Many physiologists consider that the former depends on A-fiber and the latter on C-fiber. These words, however, are not sufficient to describe perceptual characteristics of many kinds of clinical pain.

Dubuisson and Melzack (1976) showed that each of the pain syndromes caused by eight clinical diseases corresponded constellations of words which appeared in the McGill-Melzack Pain Questionnaire (MPQ) (Melzack \& Torgerson, 1971; Melzack, 1975). Grushka and Sessle (1984) showed that the words in MPQ differentiate "toothache" pain. Klepac, Dowling, and Hauge (1981) applied MPQ to laboratory pain. These studies showed that words describing pain are a reliable means of measuring pain as well as in those of other studies (Maiani \& Sanavia, 1985; Corson \& Schneider, 1984). Byrne, Troy, Bradley, Marchisello, Gersinger, van der Heide and Prieto (1982) derived four factors of the factor analysis of the responses of patients with low back pain to MPQ. These were sensory pressure, evaluative-affective-sensory, sensory-punishing affect and thermal-miscellaneous affective. The first factor was one relating to sensation itself, and explained $71.9 \%$ of the contents of pain described by the words in MPQ. The other three factors were evaluative or affective. It is a reasonable result, since $M P Q$ was designed to assess three components of pain (i.e. sensory, affective and evaluative components) and contained many affective or emotional adjectives. Turk, Rudy, and Salovey (1985) confirmed the three components by results of the factor analysis. As far as the factor analysis or the other multidimensional analysis is applied to results of MPQ, the components of affection and evaluation should appear and the sensory component should appear as a component. This sensory component does not offer details of sensory quality and intensity of pains. The factor analytical study of Japanese words of pain (Yagi, Ueda, \& Matsumoto, 1987) also showed factors containing two aspects of pain, sensation and affection and showed no factor regarding details of sensory aspect of pain. It is true that patients who feel intense pain also feel unpleasantness or fear. However, relationship between strength of experienced pain and affective expressions of the pain by language are not parallel. Affective expressions of pain depended on cultural and social learning (Zborowski, 1952). On the other hand, Melzack and Wall $(1982$, p. 61) reported " . . . the high degree of agreement on the intensity relationships among pain descriptors by subjects who have different cultural, socioeconomic and educational backgrounds, ...".

Use of words for measurement of pain has brought a confusion of the perceptual characteristics and the affective attributes of pain and brought unfortunate results for patients, since it makes affection a cause of pain rather than a result of pain. Many emotional and personality studies have been developed on the ground of this displacement.

For both patients and medical physicians or researchers, sensory aspect of pain should be differentiated from affective attribute, and characteristics of the sensory aspect should be interpreted by words common to five characteristics of other senses, such as intensity, spatial locations 
Table 1

The twenty-five words as pain descriptors

\begin{tabular}{|c|c|c|c|c|c|c|c|c|}
\hline \multicolumn{3}{|c|}{ No. Word (or phrase) } & \multirow{2}{*}{$\begin{array}{c}\text { No. } \\
9\end{array}$} & \multicolumn{2}{|c|}{ Word (or phrase) } & \multirow{2}{*}{$\begin{array}{c}\text { No. } \\
18\end{array}$} & \multicolumn{2}{|c|}{ Word (or phrase) } \\
\hline 1 & \#\# & pressing & & & rough & & \#\# & gripping \\
\hline 2 & & I feel difficulty in moving & 10 & & feel as if my body & 19 & \#\# & piercing \\
\hline 3 & \#\# & heavy & & & is stiff & 20 & & tight \\
\hline 4 & \#\# & shooting & 11 & \#\# & numbing & 21 & \# & shrinking \\
\hline 5 & & jumping & 12 & \#\# & cramping & 22 & \# & pounding \\
\hline 6 & $\# \#$ & feel as if $I$ am being cut & 13 & \# & spreading & 23 & $\# \#$ & tugging \\
\hline & & with a knife & 14 & \#\# & pulsing & 24 & & smarting \\
\hline 7 & \#\# & stabbing & 15 & \#\# & throbbing & 25 & & itching \\
\hline 8 & \#\# & feel as if something is & 16 & \#\# & prickling & & & \\
\hline
\end{tabular}

Note. The words marked by \#\# were supported by both the non-medical and medical subjects ( $p$ $<.05)$. The words marked by \# were supported by either of subjects $(p<.05)$ or the other $(p<.10)$. Details are in the text.

(area and depth of pain in a body), duration (brief or long pain), time-course (constant or intermittent) and the other characteristic such as texture in surface color or timbre in sound perception. Many people use many words as descriptors of their perceived pain. Pain descriptive words must be analyzed on the grounds of the perceptual characteristics.

Purposes of our study (see Author notes) are to clarify the perceptual characteristics of pains caused by Occupational Cervicobrachial Disorder (OCD) and similar diseases of shoulder, elbow and wrist and to compile a list of words ascertaining the perceptual characteristics of pains. In Research I, a 25-word list describing the pains was compiled. Five perceptual characteristics (duration, frequency, depth, area and strength) of the pain describing by the words were estimated and illustrated in "Pentagon-profiles". In Research II, a Japanese version of MPQ was compiled and analyzed by the same procedure in Research I. Reliability of subjects' assessments and estimations of the pains were supported on comparing both results obtained from Researches $\mathrm{I}$ and II.

\section{Research I}

\section{Method}

The 25-word list. Words in the 25-words list (Table 1) were chosen from a large number of pain-descriptive words as most fitting the pains by $O C D$ and the similar disease of the shoulder, elbow and wrist. The questions A, B, C and D (in upper half of Table 2) request information about linguistic knowledges of the words and experiences of actual pain described by the words. Five five-point scales (in lower half of Table 2) request estimates of the perceptual characteristics of pain described by the words.

Subjects. Two groups of eighteen persons each participated voluntary as subjects. One of the two groups contained in medical physicians (ten men and a woman, aged twenty-eight to forty year old) and medical students (five men and two women, twenty-three to twenty-eight) at Hamamatsu University School of Medicine. The other contained in engineering (four men), science (two men) and human science (seven men and two women) students, aged twenty-one to twenty-seven old.

Procedure. The subjects were instructed on the purpose of the research and re- 
Table 2

Questions and 5-point scales for estimating the perceptual characteristics of pain described by words in the 25-word list and the Japanese version of $M P Q$

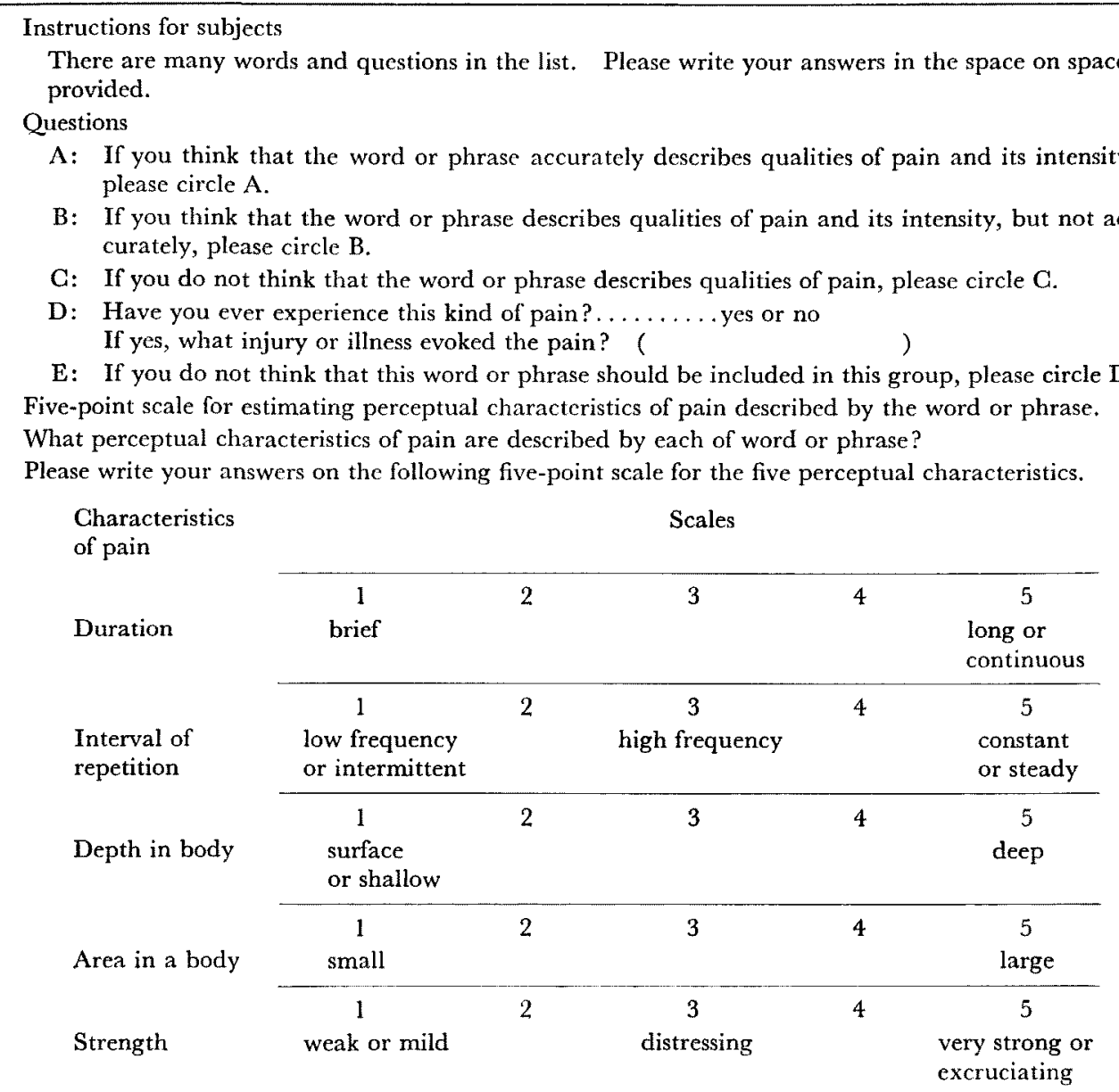

Note: 1) Question $E$ is available only for the Japanese version of MPQ in Research II. 2) The "group" means each of the twenty categories of words in MPQ (see Table 6).

quested to complete the questionnaire.

\section{Results and Discussion}

An application of the quantification method of the fourth type showed no clear clusters of the words. It is due to the fact that each of the 25 words expresses peculiar characteristics regarding of the five characteristics and sixth one (mentioned below), so no clusters appeared. We drew the pentagon-profiles of the words illustrating the five perceptual characteristics of pains by the following procedure.

On the questions $\mathrm{A}$ and $\mathrm{B}$, sixteen words marked by \#\# or \# in Table 1 were supported as descriptors of pains by majority of the subjects (thirteen of the eighteen subjects each, $p<.05$ (\#\#) for the table of the binominal distribution include three words of $p<.10$ (\#) for the non-medical subjects) who has been experienced actually the pains. On the question $\mathrm{D}$, the subjects located their experienced pains on shoulder, arm, elbow and upper half 
Table 3

Correlation coefficient $(r)$ among estimated values of the supported sixteen words in Table 1

\begin{tabular}{|c|c|c|c|c|c|}
\hline & \multicolumn{5}{|c|}{ Non-medical subjects } \\
\hline & Duration & Interval & Depth & Area & Strength \\
\hline Duration & 1.00 & $.923 * * *$ & .389 & $.614^{*}$ & .264 \\
\hline Interval & & 1.00 & .281 & $.512 *$ & .125 \\
\hline Depth & & & 1.00 & .248 & $.799 * * *$ \\
\hline \multirow[t]{2}{*}{ Area } & & & & 1.00 & .081 \\
\hline & \multicolumn{5}{|c|}{ Medical subjects } \\
\hline Duration & 1.00 & $.888 * * *$ & .225 & $.609 *$ & -.301 \\
\hline Interval & & 1.00 & .089 & $.525 *$ & -.283 \\
\hline Depth & & & 1.00 & -.050 & $.549 *$ \\
\hline Area & & & & 1.00 & $-.629 * *$ \\
\hline
\end{tabular}

Table 4

The perceptual characteristics of the supported sixteen words in Table 1 marked by \#\# or \#

\begin{tabular}{|c|c|c|c|c|c|c|c|c|c|}
\hline \multicolumn{4}{|c|}{ Non-medical } & & & \multicolumn{4}{|c|}{ Medical } \\
\hline $\begin{array}{l}\text { Time } \\
1 \& 2^{*}\end{array}$ & Depth & $\begin{array}{c}\text { Area } \\
4^{*}\end{array}$ & $\begin{array}{l}\text { Strength } \\
5^{*}\end{array}$ & & No. word & $\operatorname{Time}_{1 \& 2 *}$ & $\underset{3^{*}}{\text { Depth }}$ & $\underset{4 *}{\text { Area }}$ & $\begin{array}{c}\text { Strength } \\
5^{*}\end{array}$ \\
\hline H & 世 & $H$ & $H$ & 15 & throbbing & \# & $H$ & $H$ & $H$ \\
\hline H & 世 & $H$ & $H$ & 8 & $\begin{array}{l}\text { as if something is } \\
\text { biting into me }\end{array}$ & $H$ & $H$ & H & H \\
\hline H & \# & $H$ & $H$ & 14 & pulsing & \# & H & $H$ & $H$ \\
\hline \# & $H$ & $H$ & $H$ & 1 & pressing & H & $H$ & $H$ & $H$ \\
\hline H & $H$ & 世 & $H$ & 13 & spreading & $H$ & H & H & + \\
\hline H & $H$ & $H$ & H & 3 & heavy & 卅 & H & H & $H$ \\
\hline H & $H$ & $H$ & $H$ & 7 & stabbing & H & H & $H$ & $H$ \\
\hline 世 & + & $H$ & $H$ & 23 & tugging & W & + & $H$ & + \\
\hline$H$ & H & H & $H$ & 22 & pounding & H & H & $H$ & + \\
\hline H & $H$ & $H$ & $H$ & 12 & cramping & H & $H$ & H & H \\
\hline$H$ & $H$ & $H$ & $H$ & 21 & shrinking & H & H & $H$ & $H$ \\
\hline$H$ & $H$ & $H$ & $H$ & 19 & piercing & $H$ & $H$ & + & $H$ \\
\hline H & + & $H$ & + & 11 & numbing & $H$ & H & $H$ & + \\
\hline$H$ & + & + & + & 16 & prickling & $H$ & + & + & + \\
\hline+ & $H$ & + & $H$ & 6 & $\begin{array}{l}\text { as if I am being } \\
\text { cut with a knife }\end{array}$ & H & H & + & H \\
\hline+ & H & + & $H$ & 4 & shooting & + & H & + & H \\
\hline
\end{tabular}

Note. 1) Mark \# shows long duration, short interval, deep, large area and strong of pain. Mark + shows short duration, long interval, surface or shallow and small area and weak of pain. Mark $\#$ shows medium of these. 2) Numerals marked by $*, 1+2,3,4$ and 5 correspond to the five scales in Table 2 and to five axes of the pentagons in Fig. 1. The axis upper centered correspond to Scale 1. The other four axes correspond 2 to 5 scales clockwise, so the left axis is Scale 5.

on their bodys, and described several kinds of symptoms; stiff shoulder (KATAKORI) or back pain, sprain, contusion, sunburn, neuralgia, wound, inflammation and headache. So these sixteen words were supported as descriptors of the pains caused OGD and the similar diseases. The other nine words of the 25 words were 
Table 5

Correlation coefficient $(r)$ among estimated value of the supported thirty-five words

\begin{tabular}{|c|c|c|c|c|c|}
\hline & \multicolumn{5}{|c|}{ Non-medical subjects } \\
\hline & Duration & Interval & Depth & Area & Strength \\
\hline Duration & 1.00 & $.871 * * *$ & $.561 * * *$ & $.610 * * *$ & $.357 *$ \\
\hline Interval & & 1.00 & $.595 * * *$ & $.697 * * *$ & $.392 *$ \\
\hline Depth & & & 1.00 & $.544 * * *$ & $.747 * * *$ \\
\hline \multirow[t]{2}{*}{ Area } & & & & 1.00 & .332 \\
\hline & \multicolumn{5}{|c|}{ Medical subjects } \\
\hline Duration & 1.00 & $.939 * * *$ & $.385 *$ & $.481 * *$ & .279 \\
\hline Interval & & 1.00 & .309 & $.393^{*}$ & .265 \\
\hline Depth & & & 1.00 & .288 & $.752 * * *$ \\
\hline Area & & & & 1.00 & .278 \\
\hline
\end{tabular}

rejected from calculations for Tables 3 and 4 , since validity of these words as pain descriptors were lower than those of the sixteen words because the nine words were supported by minority of the subjects.

Differences of the means among the five characteristics and in both the non-medical and medical subjects were not significant by the analysis of variance of the two factor design. Table 3 shows correlation coefficients $(r)$ of the estimated values between each two pair of the five scales. High correlations appeared in four pairs: Duration and Interval; Depth and Strength; Area and Duration; and Area and Interval. These high correlations show the followings: A pain of long duration is repeated; a deep pain is strong; and a pain of large area in the body is prolonged and repeated.

Table 4 shows the perceptual characteristics of the sixteen supported words. The symbols $H$ in Table 5 identify the high range (3.55-5.00) of the average estimated value of each scale. Symbol H shows medium range (3.54-2.55), symbol + shows low range (2.54-1.00). In the second row "Time" is a combination of two scales, Duration and Interval, since these two scales were highly correlated (in Table 3). "Depth", "Area" and "Strength" correspond to those of the five scales. In Table 5 the pains described by the sixteen supported words were arranged top to bottom on a rank order of Time. The pains on the same rank of order of Time were arranged by an order of Depth. Then, in the same rank of Depth, Strength is arranged. Area is finally arranged. Duration, interval and depth are important characteristics of pain, since it observed empirically that patients tolerate brief pain more than long or constant pain, even if the strength of the brief pain is more than that of the long or constant pain. A pain repeated frequently is felt more strongly than a low frequency pain even when the strength of these two pains are equal. It also observed empirically that patients also tolerate surface pain more than deep pain, even if the strengths of the two pains are same. So, in Table 5, it ought to be said the pains are arranged on rank order of "intolerability" or "seriousness" of the pain.

In Fig. 1 the pentagon-profiles illustrate the five characteristics of the pains clearer than in Table 5. The pentagons in figures were arranged left to right in each row on the rank order of the intolerability as those in Table 5. These pentagons were drawn by the following procedure. The radius $(R)$ of the outer circle of the pentagons is 
calculated by the following Equation (1),

$$
R=E \max -E \min
$$

$E \max =5 \times \mathcal{N}, E \min =1 \times \mathcal{N}$,

$5=$ maximum value of the five point on a given scale,

$1=$ minimum value of the five point on

a given scale,

$\mathcal{N}=$ number of subjects who supported a given word.

The radius of the inner circle is one half of the radius of the outer circle. The length of each of the five axes (from center of the inner circle to periphery of the outer's) a given pentagon corresponded to each value $\left(E_{0}\right)$ of the five scales calculated by the Equation (2).

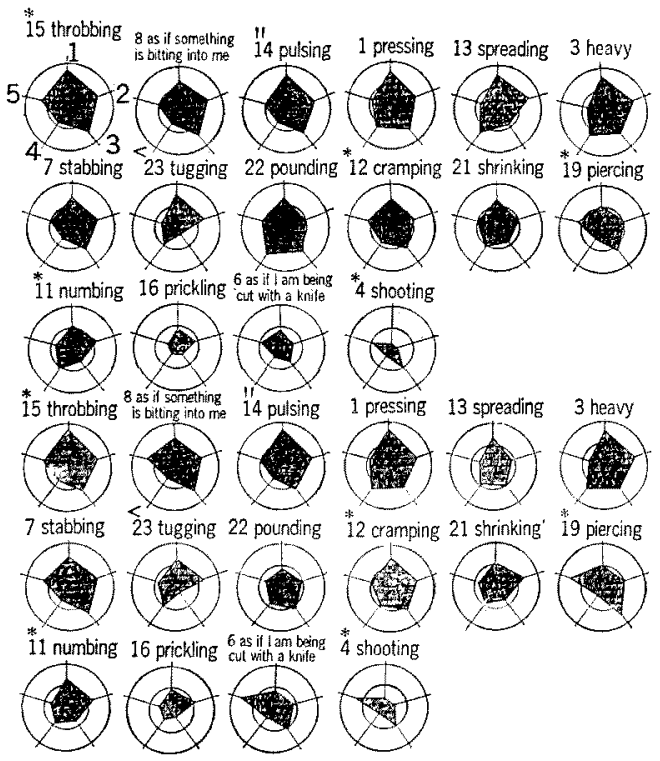

Fig. 1. The pentagon-profiles of the pain described by the supported sixteen word in Table 5 . The pentagons are arranged left to right in each row and top row to bottom row. Numerals 1 to 5 , around a pentagon, correspond to the five scales. Numerals 1 shows Duration, 2 shows Interval and 3, 4 and 5 correspond to Depth, Area and Strength. The pentagons in upper half in Figure are nonmedical subjects', and in lower half are medical subjects'. The words with symbol * are the same as those in Figs. 2 and 3. Details about symbols " and $<$ are in the text.

$$
E_{0}=\sum_{i=1}^{5}(\text { estimated value } \times n) / E \max
$$

estimated value $=\mathbf{a}$ given value assigned on a given scale,

$i=$ each of estimated value of the five points ( 1 to 5 ) on the given scale, $n=$ number of subjects who assigned the estimated value on the given scale.

The length of each axis of a given pentagon is relatively corresponded to the marks $H$, $\#$ or + in Table 5. Larger pentagons illustrate more intolerable pain than smaller pentagons. Profiles of the pentagons indicate relative relationship among amounts of the estimated values for the five characteristics of pain. Similarity of profiles among the pentagons indicates relative similarity of pains regarding the estimated five characteristics.

In Table 5 and Fig. 1, all estimations of the pains by both the non-medical and medical subjects were very similar except nos. $8,19,6$ and 4 . The medical subjects estimated these four pains more intense than those by the non-medical subjects. It is due to the fact that the medical subjects know empirically many kinds of intolerable pain of patients, so they assessed higher values for these pain than by the non-medical subjects.

Most intolerable pains were nos. 15, 8, 14, 13, 3, 7 and 23 (except the medical subjects' estimation of no. 13). These pains were repeated frequently with long duration and located deep or large area in a body. Most tolerable pains were nos. 6 and 4. These were brief pain with small area. The others, nos. 22, 12, 21, 19,11 and 16 were medium pains regarding all five characteristics.

Finally, the sixth characteristic of the pains is discussed. This characteristic expressed in meaning of the words and does not express in the pentagons. This is similar to textures in surface color. Two colors of equal hue, equal saturation and equal lightness are perceived as two different colors, if their textures are differ from each other. A pair of pentagons, 
nos. 15 and 14 for both subjects were very similar to each other. If these pains are perceived one after another, patients might feel similarity of the five characteristics and feel difference in rhythmical change of intensity of the pains. This difference related to the sixth characteristic. A meaning (in Japanese) common between both word "throbbing" (no. 15) and "pulsing" (no. 14) expresses " rhythmical change" of strength of pains. The pentagons could not illustrate the rhythm. "Throbbing" expresses slow rhythm and "pulsing" expresses faster rhythm slightly than that of "throbbing". It observed empirically that patients claim their perceived pain of suppurative disorders or OCD by these words. "Tugging" (no. 33) and "Pricking" (no. 16) expresses most fast rhythm. "Shooting" (no. 4) describes something running or moving rapidly (persons, animals or cars). Word shooting pain is frequently used by patients to express a kind of strong pain with short duration caused by moving of arm which is suffered from OCD or neuralgia. The shooting pain also occurs by striking one's elbow against a rock or a hard board (and occurs spreading pain at the same time). The profile of pentagon of no. 4 does not express this "moving" characteristic. "Biting" (no. 8) and "stabbing" (no. 7) describe something into a body "deeper and deeper". "Spreading" expresses that something is spreading "gradually". Meanings of the other words also express the sixth characteristics of the pains. The meanings of the pain-descriptive words are fruitful for medical physicians or researchers to recognize pains expressed verbally by patients or subjects as well as mentioned by Melzack (1975). However, Melzack and other researchers have not clarified the perceptual characteristics. The sixteen words can be described the pains caused by OCD and similar disease.

\section{Research II}

\section{Method}

A Japanese version of MPQ was compiled. Questions and scales were the same as those for the 25 words in Table 2. The same subjects who participated in Research I completed the questionnaire by the same procedure as in Research $I$.

\section{Results and Discussion}

All tables and figures in Research II were drawn by the same procedure as those for tables and figure in Research I. Thirty-five words shown in these tables and figures were categorized into the question $\mathrm{A}$ or $\mathrm{B}$ by majority $(p<.05$ for the table of binomial distribution) of both the non-medical and medical subjects who has been experienced actually these pain. Locations and symptoms of these experienced pains were more than those in the results of Research I. Chest pain, fracture, laceration, stomachache and toothache appeared. These thirty-five words were supported as descriptors of pains not only caused by OCD and the similar diseases, but also by the other pains located on head or lower half in a body. It is reasonable results that $\mathrm{MPQ}$ was compiled for assessing many kinds of pains. The other nineteen of the seventy-eight words were supported by minority of the subjects as pain descriptors. Validity of the nineteen words as pain descriptors was lower than the thirty-five words. The other twenty-four of the seventy-eight words were categorized into the question $\mathrm{C}$ as no-pain descriptors. Both the eighteen and the twenty-four words were rejected from calculations for tables.

Differences of the means among the five characteristics and in both the non-medical and medical subjects were not significant by the analysis of variance of the two factor design. In Table 5 four highest correlations appeared between Duration and Interval; Depth and Strength; Area 
Table 6

The perceptual characteristics of the supported thirty-five words in MPQ

\begin{tabular}{|c|c|c|c|c|c|c|c|c|c|}
\hline \multicolumn{6}{|c|}{ Non-medical } & \multicolumn{4}{|c|}{ Medical } \\
\hline Time & Depth & Area & Strength & No. & Word & Time & Depth & Area & Strength \\
\hline $\mathrm{H}$ & $\mathrm{H}$ & $H$ & \# & $6(1-6)$ & pounding & \# & $H$ & $H$ & 州 \\
\hline H & H & $H$ & \# & $5(1-5)$ & beating & $H$ & H & $H$ & $H$ \\
\hline$H$ & $\mathrm{H}$ & $H$ & $\mathrm{H}$ & $42(10-4)$ & splitting & $\#$ & \# & $H$ & $\#$ \\
\hline H & H & $H$ & H & $16(4-2)$ & cutting & $H$ & $H$ & $H$ & $H$ \\
\hline H & H & $H$ & $H$ & $17(4-3)$ & lacerating & H & $H$ & H & \# \\
\hline H & H & $H$ & $H$ & $22(5-5)$ & crushing & H & H & $H$ & $H$ \\
\hline H & H & H & $H$ & $33(8-4)$ & stinging & 世 & $H$ & + & $H$ \\
\hline$H$ & H & $H$ & $H$ & $21(5-4)$ & cramping & $H$ & $H$ & $H$ & $H$ \\
\hline$\#$ & $H$ & + & $H$ & $37(9-4)$ & aching & H & $H$ & + & + \\
\hline$\#$ & $H$ & H & H & $4(1-4)$ & throbbing & H & $H$ & + & $H$ \\
\hline H & $H$ & $H$ & H & $27(7-2)$ & burning & $H$ & + & $H$ & $H$ \\
\hline$H$ & $H$ & + & $H$ & $15(4-1)$ & sharp & + & + & + & $H$ \\
\hline H & $H$ & H & + & $19(5-2)$ & pressing & $H$ & $H$ & $H$ & $H$ \\
\hline$\#$ & $H$ & $H$ & + & $26(7-1)$ & hot & H & + & $H$ & $H$ \\
\hline$H$ & + & H & H & $28(7-3)$ & scalding & \# & + & $H$ & $H$ \\
\hline \# & + & $H$ & H & $30(8-1)$ & tingling & $H$ & + & + & + \\
\hline H & $H$ & $H$ & + & $48(18-2)$ & numb & $H$ & + & $H$ & + \\
\hline$H$ & \# & + & \# & $14(3-5)$ & lancinating & H & \# & $H$ & \# \\
\hline$H$ & $H$ & $H$ & H & $51(18-5)$ & tearing & $H$ & $H$ & $H$ & $H$ \\
\hline$H$ & $H$ & + & H & $25(6-3)$ & wrenching & $H$ & $H$ & + & H \\
\hline$H$ & $H$ & + & H & $13(3-4)$ & stabbing & H & 卅 & $H$ & H \\
\hline$H$ & $H$ & $H$ & $H$ & $50(18-4)$ & squeezing & $H$ & H & $H$ & $H$ \\
\hline$H$ & H & + & $H$ & $12(3-3)$ & drilling & H & $H$ & $H$ & H \\
\hline$H$ & H & + & $H$ & $46(17-4)$ & piercing & H & \# & + & H \\
\hline$H$ & $H$ & + & $H$ & $11(3-2)$ & boring & $H$ & H & + & $H$ \\
\hline$H$ & H & + & $H$ & $20(5-3)$ & gnawing & $H$ & $\#$ & + & $H$ \\
\hline$H$ & $H$ & + & + & $8(2-2)$ & flashing & $H$ & $H$ & + & + \\
\hline$H$ & + & $H$ & $H$ & $3(1-3)$ & pulsing & $H$ & + & + & $H$ \\
\hline$H$ & + & + & + & $23(6-1)$ & tugging & $H$ & $H$ & $H$ & $H$ \\
\hline$H$ & + & + & + & $2(1-2)$ & quivering & $H$ & + & + & + \\
\hline$H$ & + & + & + & $1(1-1)$ & flickering & H & + & + & + \\
\hline+ & H & $H$ & H & $9(2-3)$ & shooting & + & $H$ & + & $H$ \\
\hline+ & + & + & + & $39(10-1)$ & tender & + & + & + & $H$ \\
\hline+ & + & + & + & $10(3-1)$ & pricking & + & + & + & $H$ \\
\hline+ & + & + & + & $32(8-3)$ & smarting & + & + & + & $H$ \\
\hline
\end{tabular}

Note. 1) Symbol \# shows long duration, short interval, deep, large area and strong of pain. Symbol + shows short duration, long interval, shallow or surface and small area and weak of pain. Symbol H shows medium of these. 2) Numerals written in parentheses are number of the category (left) and number of the word (right) in MPQ. 3) The other details are the same as those in Table 4.

and Interval, as well as those in Table 3.

In Table 6 and Figs. 2 and 3, all estimations of the pains by both the nonmedical and medical subjects were very similar except nos. 50, 12, 46 and 11 . The medical subjects estimated these four pains stronger and deeper than those by the non-medical subjects. It was also found in Table 4 (nos. 8, 19,6 and 4). Most intolerable pains were nos. 6, 5, 42, $16,17,22,33,21,37,50$ and 12 (except nos. 50 and 12 for the non-medical sub- 
jects, and except medicals' 37). Most tolerable pains were nos. $9,39,10$ and 32 . The others were medium pains.

Comparing arrangement of the words in Table 6 and Figs. 2 and 3 with those in $M P Q$, several words were arranged in different places. It is reasonable results. The words in MPQ were arranged from weak to strong pain in each categories. These were defined by Melzack and Torgerson (1971) and shown by the numerals written in the parentheses in Table 6. On the other hand, in Table 6 and Figs. 2 and 3 the words were arranged by the rank order of the intolerability.

Regarding the sixth characteristic three words, "throbbing" (no. 5), " beating" (no. 4) and "stinging" (no. 33) include a meaning, rhythmical change of strength of pains, common among these word. The other three; " tingling" (no. 30), "quiv-

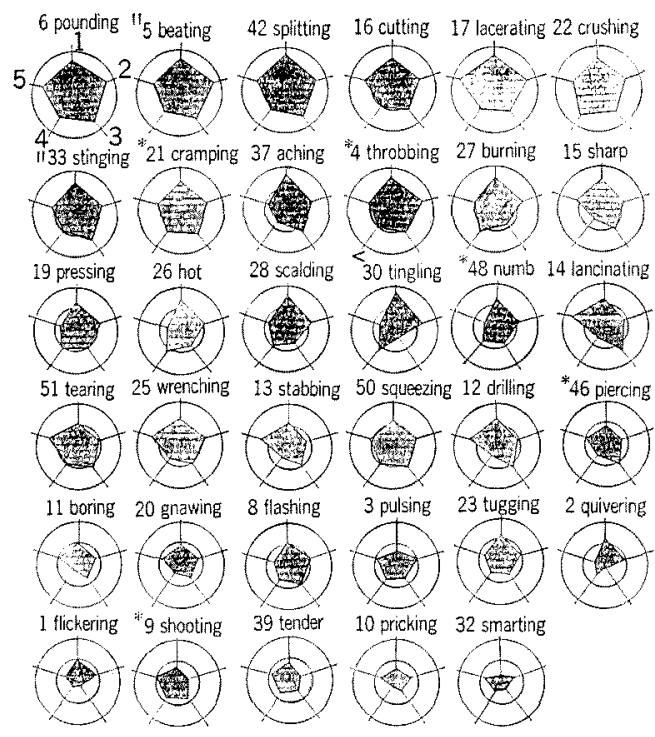

Fig. 2. The pentagon-profile of pain described by the thirty-five words supported by non-medical subjects in Table 6. The pentagons are arranged left to right in each row and top row to bottom row on rank order of the intolerability. Numerals 1 shows Duration, 2 shows Interval and 3, 4 and 5 correspond to Depth, Area and Strength. The words with symbol * are the same as those in Fig. 1. Details of symbols " and $<$ are in the text. ering" (no. 2) and "flickering" (no. 1) expressed faster rhythm than those of the three words above. Details of "shooting " (no. 9) were explained in the results of Research I. Meanings of the other words express the sixth characteristics of pains. Meanings of the pain-descriptive words are fruitful for recognizing the pains.

\section{Reliability of the subjects' verbal reports}

Five words and two pairs of synonyms were used common both in the 25-words list and in MPQ. The five words were "shooting" (no. 4 in the 25 words and no. 9 (2-3) in MPQ), "numbing" (no. 1 and no. 48 (18-2)), "cramping" (no. 12 and no. 21 (5-4)), "throbbing" (no. 15 and no. $5(1-5)$ ) and "piercing" (no. 19 and no. $46(17-4))$. These were marked by $*$ in Figs. 1, 2 and 3 . Profiles of pentagons of these five words in figures were very similar, although words were presented to the subjects in different context.

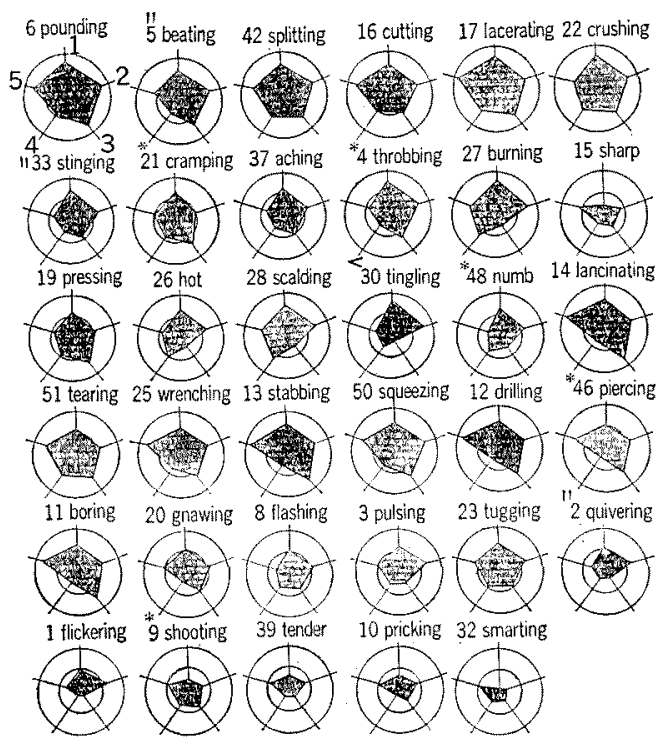

Fig. 3. The pentagon-profile of the pain described the thirty-five words supported by medical subjects. Arrangement of the pentagons, details of numerals and symbols are the same as those in Fig. 2. 
The words in the 25-words list were arranged by Japanese alphabetical order. The words in MPQ were arranged by categorical order in peculiar to $\mathrm{MPQ}$. The subjects did not influenced by the context effect. Reliability of the subjects' estimation was supported.

One of the two pairs of synonyms was "tugging" (in Fig. 1, no. 23, marked by $<$ ) and "tingling" (in Figs. 2 and 3, no. 30). The other was "pulsing" (in Fig. 1 , no. 14 marked by ") and "stinging" (in Figs. 2 and 3 marked by "). Furthermore, in the Japanese version of MPQ, "beating" (in Figs. 2 and 3, no. 5) was also a synonym of throbbing. Each pair of these synonyms showed similar profiles. The reliability was also supported by the profiles of the synonyms.

In psychophysical researches, it is believed that there is no reliability in estimations of stimuli presented by words, since subjects respond to their own images of stimuli as evoked by the words. However, our results shows the reliability. In our prior work (Satow, 1983) has been showed already the test-retest reliability obtained from large sample of subjects (120 men participated). Correlation $(r)$ of the reliability was average $r .541$, (from .308 to .726) for the 20-item checklist. Furthermore, in Satow (1986, 1987), factor stability were shown in factor analytical studies of the 60-item check list requested estimating environmental sensory stimuli.

\section{Conclusions}

1) The sixteen words in Table 4 and the thirty-five words in Table 6 were supported as the pain-descriptors caused by OCD and the similar diseases by both the nonmedical and medical subjects who experienced these pains. Some of the thirty-five words, however, were supported as the descriptors of the other several kinds of pain. 2) The supported words differentiated perceptual characteristics from emotional or evaluative attribute of pains. The five perceptual characteristics of the pains described by words supported were illustrated in the pentagon-profiles (in Figs. 1, 2 and 3). 3) In the tables and the figures pains were arranged on the rank order of the intolerability. Larger pentagons look visually illustrated intolerable pain more than smaller pentagons. Similarity of profiles of the pentagons, regardless larger or smaller, showed relative similarity among the five characteristics of the pain. 4) The sixth characteristic was expressed in meanings of the words. This characteristic is fruitful for recognizing the pains. 5) Reliability of subjects' estimations was supported.

\section{References}

Byrne, M., Troy, A., Bradley, L. A., Marchisello, P. J., Gersinger, K. F., van der Heide, L. H., \& Prieto, E.J. 1982 Cross-validation of the factor structure of the McGill Pain Questionnaire. Pain, 13, 193-201.

Corson, J. A., \& Schneider, M. J. 1984 The Dartmonth Pain Questionnaire: An adjunct to the McGill Pain Questionnaire. Pain, 19, 5968.

Dubuisson, D., \& Melzack, R. 1976 Classification of clinical pain descriptors by multiple group discriminant analysis. Experimental Neurology, 51, 480-487.

Grushka, M., \& Sessle, B. J. 1984 Applicability of the McGill Pain Questionnaire to the differentiation of 'toothache' pain. Pain, 19, 4857.

Klepac, R. K., Dowling, J., \& Hauge, G. 1981 Sensitivity of the McGill pain questionnaire to intensity and quality of laboratory pain. Pain, 10, 199-207.

Maiani, G., \& Sanavia, E. 1985 Semantics of pain in Italy: the Italian version of the McGill Pain Questionnaire. Pain, 22, 399-405.

Melzack, R. 1975 The McGill pain questionnaire: Major properties and scoring methods. Pain, 1, 277-299.

Melzack, R., \& Torgerson, W. S. 1971 On the language of pain. Anesthesiology, 34, 50-59.

Melzack, R., \& Wall, P. D. 1982 The challenge of pain. New York: Penguin Books. 
Mountcastle, V. B. 1980 Pain and temperature sensibilities. In V. B. Mountcastle (Ed.), Medical physiology (Chap. 13). London: The G.V. Mosby Co. Pp. 391-427.

Satow, A. 1983 Nonparallel relationship between tolerance and sensitivity for visual and auditory environmental stimuli. Journal of Light F? Visual Environment, 7, 86-92.

Satow, A. 1986 An ecological approach to mechanisms determining individual differences in perception. Perceptual and Motor Skills, 62, 983-998.

Satow, A. 1987 Four properties common among perceptions confirmed by a large sample of subjects: An ecological approach to mechanisms of individual differences in perception: II. Perceptual and Motor Skills, 64, 507-520.
Schmidt, R. F. 1978 Fundamentals of sensory physiology. New York: Springer-Verlag.

Turk, D. C., Rudy, T. E., \& Salovey, P. 1985 The McGill Pain Questionnaire reconsidered: confirming the factor structure and examining appropriate uses. Pain, 21, 385-397.

Wolff, B. B. 1978 Behavioral measurement of pain. In R. A. Sternback (Ed.), The psychology of pain New York: Raven Press. Pp. 129-168.

Yagi, T., Ueda, M., \& Matsumoto, S. 1987 Analysis of Pain-descriptive words: VI. $A n$ nual report of Shiraumegakuen College, 23, 63-72. (In Japanese)

Zborowski, M. 1952 Gultural components in responses to pain. Journal of Social Issues, 8, 16-30. (Received Nov. 2, 1987; accepted June 4, 1988) 